01

\title{
Широкополосные хаотические колебания в слабосвязанном ансамбле автоколебательных осцилляторов
}

\author{
(C) Н.В. Станкевич ${ }^{1-3}$, Е.С. Попова ${ }^{1,2}$, А.П. Кузнецов ${ }^{2}$, Е.П. Селезнев ${ }^{2,4}$ \\ ${ }^{1}$ Саратовский государственный технический университет им. Гагарина Ю.А., Саратов, Россия \\ ${ }^{2}$ Саратовский филиал Института радиотехники и электроники им. В.А. Котельникова РАН, Саратов, Россия \\ ${ }^{3}$ Санкт-Петербургский государственный университет, Санкт-Петербург, Россия \\ ${ }^{4}$ Саратовский национальный исследовательский государственный университет им. Н.Г. Чернышевского, Саратов, Россия \\ E-mail: stankevichnv@mail.ru
}

Поступило в Редакцию 8 июля 2019г.

В окончательной редакции 8 июля 2019г.

Принято к публикации 17 сентября 2019г.

\begin{abstract}
Представлено численное исследование связанных в кольцо осцилляторов ван дер Поля. Проведено исследование особенностей хаотической динамики с помощью анализа спектров Фурье и спектра показателей Ляпунова. Показано, что хаотическая динамика, возникающая при разрушении многочастотных торов, обладает широкополосным спектром.
\end{abstract}

Ключевые слова: ансамбли взаимодействующих осцилляторов, хаотические колебания, показатели Ляпунова, широкополосность.

DOI: 10.21883/PJTF.2019.24.48796.17967

Одним из перспективных направлений прикладного использования радиофизических генераторов являются системы скрытой коммуникации на базе синхронизации хаотических сигналов [1-3]. Такого рода задачи предъявляют определенные требования к генераторам сложных сигналов. Одним из важнейших свойств является широкополосность генерируемого сигнала. Свойство широкополосности может быть связано со сценарием, в результате которого формируется хаотический сигнал. Так, например, при формировании хаотического сигнала в результате разрушения многочастотных торов спектр будет содержать компоненты разрушенных торов. В данном контексте можно вспомнить о сценарии Ландау-Хопфа, когда хаотическая динамика возникает в результате вовлечения большего числа частотных компонент [4]. Наиболее удобными моделями для реализации устойчивых многочастотных квазипериодических колебаний являются многоконтурные генераторы [5] или ансамбли взаимодействующих автоколебательных систем [6,7]. В работе [6] была разработана модель, представляющая собой ансамбль связанных осцилляторов ван дер Поля, в котором наблюдалась последовательность квазипериодических бифуркаций. Однако в такой модели области хаотической динамики в пространстве параметров малы. В рамках настоящей работы предлагается исследование ансамбля связанных осцилляторов ван дер Поля, в котором наблюдается формирование хаоса в результате разрушения многочастотного тора, и особое внимание уделено спектральным характеристикам хаотических аттракторов.

Объектом исследования настоящей работы является ансамбль взаимодействующих осцилляторов ван дер Поля со связью типа „кольцо“, математическую модель которого можно записать в следующем виде:

$$
\begin{gathered}
\ddot{x}-\left(\lambda_{1}-x^{2}\right) \dot{x}+x+\frac{\mu}{2}(2 x-w-y)=0, \\
\ddot{y}-\left(\lambda_{2}-y^{2}\right) \dot{y}+\left(1+\frac{\Delta}{4}\right) y+\frac{\mu}{2}(2 y-x-z)=0 \\
\ddot{z}-\left(\lambda_{3}-z^{2}\right) \dot{z}+\left(1+\frac{\Delta}{2}\right) z+\frac{\mu}{2}(2 z-y-v)=0, \\
\ddot{v}-\left(\lambda_{4}-v^{2}\right) \dot{v}+\left(1+\frac{3 \Delta}{4}\right) v+\frac{\mu}{2}(2 v-z-w)=0, \\
\ddot{w}-\left(\lambda_{5}-w^{2}\right) \dot{w}+(1+\Delta) w+\frac{\mu}{2}(2 w-v-x)=0 .
\end{gathered}
$$

Здесь $x, y, z, v, w$ - динамические переменные осцилляторов, $\lambda_{i}$ - параметры, отвечающие за возбуждение автоколебаний в каждом индивидуальном осцилляторе ван дер Поля, $\Delta-$ частотная расстройка между осцилляторами, $\mu-$ коэффициент связи. В работе [6] рассматривался подобный ансамбль, только с глобальной связью. Частоты генераторов распределены эквидистантно по кольцу. Обязательным условием для возникновения последовательности квазипериодических бифуркаций Хопфа была неидентичность по параметрам возбуждения $\lambda_{i}$. В случае идентичности по параметрам возбуждения в системе возникают вырождения, и таким образом можно сохранить в пространстве параметров области многочастотных квазипериодических колебаний. В нашей работе мы зафиксируем идентичными все параметры возбуждения $\lambda_{i}=\lambda=0.5$ и рассмотрим особенности динамики системы при малой силе связи и вариации параметра частотной расстройки.

Основной инструментарий, позволяющий идентифицировать сложные колебательные режимы, включая 
Таблица 1. Список возможных динамических режимов, соответствие их сигнатуры спектра показателей Ляпунова и обозначения

\begin{tabular}{c|c|c}
\hline Режим & $\begin{array}{c}\text { Сигнатура спектра } \\
\text { показателей Ляпунова }\end{array}$ & Обозначение \\
\hline Периодический & $(0,-,-,-,-,-,-,-,-)$ & 1 \\
Двухчастотный квазипериодический & $(0,0,-,-,-,-,-,-)$, & 2 \\
Трехчастотный квазипериодический & $(0,0,0,-,-,-,-,-)$, & 3 \\
Четырехчастотный квазипериодический & $(0,0,0,0,-,-,-,-,-)$ & 4 \\
Пятичастотный квазипериодический & $(0,0,0,0,0,-,-,-,-)$ & 5 \\
Хаос & $(+, 0,-,-,-,-,-,-)$, & $c 1$ \\
Хаос с одним дополнительным нулевым & $(+, 0,0,-,-,-,-,-)$, & $c 2$ \\
показателем Ляпунова & $(+, 0,0,0,-,-,-,-,-)$, & $c 3$ \\
Хаос с двумя дополнительными нулевыми & & \\
показателями Ляпунова & $(+,+, 0,-,-,-,-,-,-)$, & $c 4$
\end{tabular}

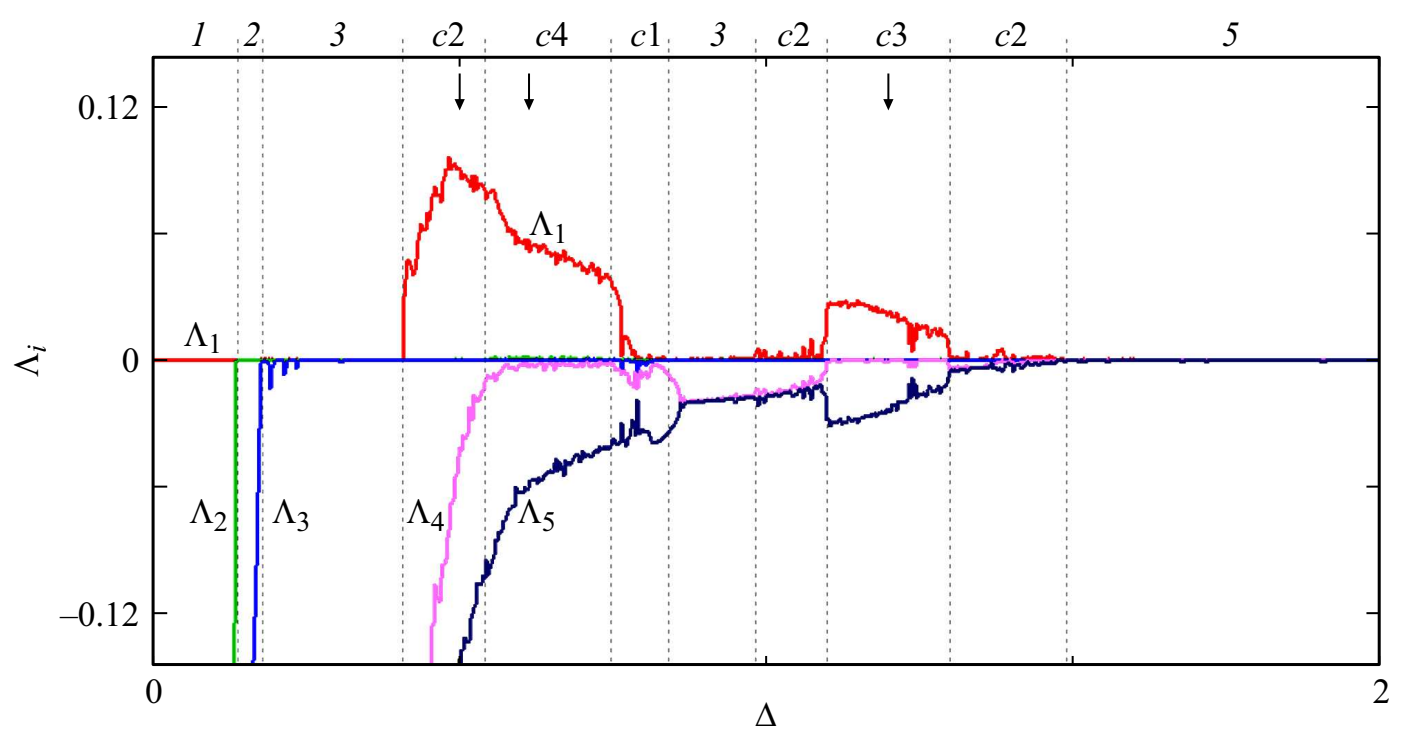

Рис. 1. Зависимости пяти старших показателей Ляпунова ансамбля взаимодействующих осцилляторов ван дер Поля (1) при $\lambda=0.5, \mu=0.12$. Пунктирными линиями отмечены области различных динамических режимов в соответствии с обозначениями, приведенными в табл. 1.

хаос, гиперхаос, и классифицировать многочастотные квазипериодические режимы, - это спектр показателей Ляпунова. Для модели (1) был рассчитан спектр показателей Ляпунова с помощью алгоритма Бенеттина и ортогонализации по Грамму-Шмидту.

Поскольку сложные колебательные режимы и квазипериодические колебания с различным числом частотных компонент сохраняются только при малой силе связи взаимодействия в ансамбле, мы зафиксировали этот параметр как $\mu=0.12$. На рис. 1 представлен однопараметрический график зависимости пяти старших показателей Ляпунова при вариации частотной расстройки между осцилляторами в ансамбле (оставшиеся пять показателей всегда отрицательны). На представленном графике по сигнатуре спектра показателей Ляпунова можно выделить девять типов динамического поведения, которые детально описаны в табл. 1. При малой частот- ной расстройке реализуются периодические колебания. При небольшом увеличении частотной расстройки наблюдаются две бифуркации седло-узел, в результате которых происходит переход от периодических колебаний к трехчастотным квазипериодическим. После этого наблюдается разрушение трехчастотного тора с формированием хаотической динамики. Хаос, возникший в результате разрушения высокочастотных торов, обладает своей спецификой: в спектре показателей Ляпунова такого режима могут присутствовать дополнительные нулевые экспоненты, отвечающие за нейтральные направления, сохранившиеся при разрушении высокочастотных торов. Таким образом, в результате разрушения трехчастотного тора в ансамбле (1) формируется хаос с одним положительным и двумя нулевыми показателями Ляпунова. При дальнейшем увеличении параметра $\Delta$, отвечающего за частотную расстройку, одна из нуле- 

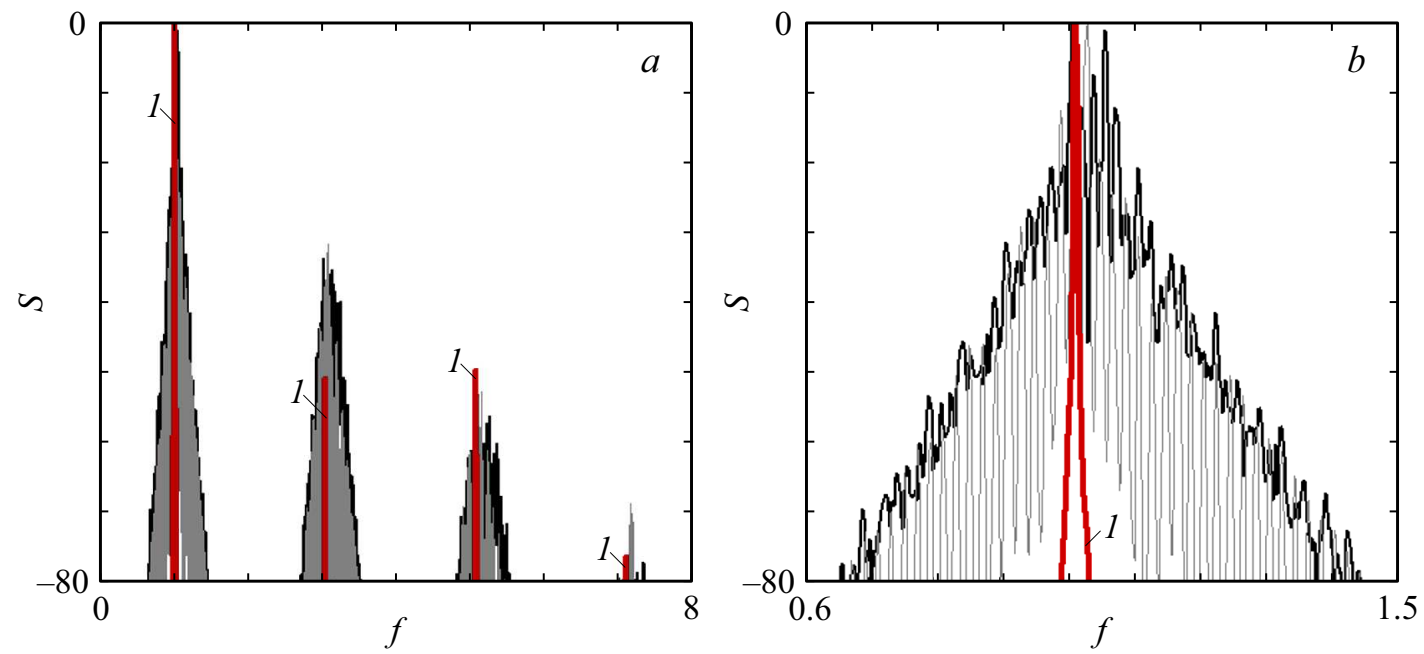

Рис. 2. Спектры Фурье ансамбля взаимодействующих осцилляторов ван дер Поля $(1), \lambda=0.5, \mu=0.12$, при переходе от периодических колебаний к трехчастотным квазипериодическим: $\Delta=0.12-$ периодический режим (линия 1$) ; \Delta=0.15-$ двухчастотный квазипериодический режим (обозначено серым); $\Delta=0.2-$ трехчастотный квазипериодический режим (обозначено черным). $a$ - полный спектр, $b-$ увеличенный фрагмент.
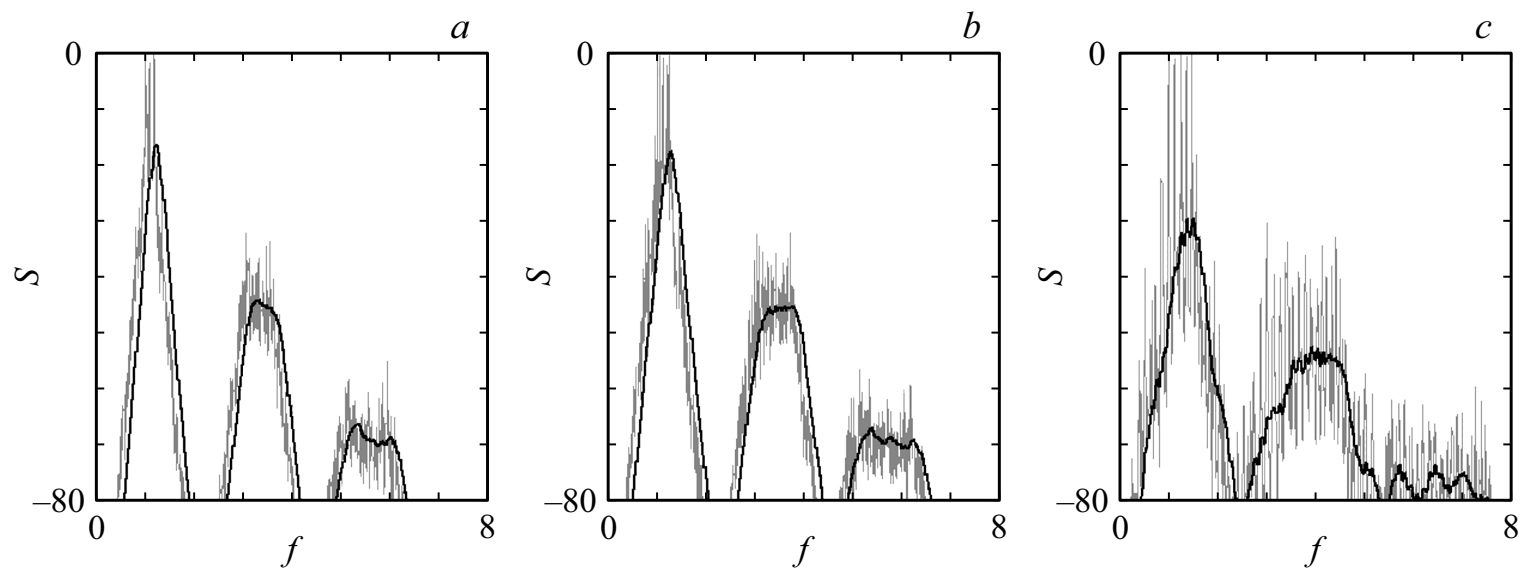

Рис. 3. Спектры Фурье ансамбля взаимодействующих осцилляторов ван дер Поля (1) для различных хаотических режимов при $\lambda=0.5 . \mu=0.12 . \Delta=0.5(a), 0.61(b)$ и $1.2(c)$. Соответствующие значения параметров указаны стрелками на рис. 1 .

вых экспонент становится положительной, и в системе наблюдается гиперхаос. Далее наблюдается переход к хаосу с одним положительным и одним нулевым показателем, который при дальнейшем увеличении частотной расстройки трансформируется в трехчастотные квазипериодические колебания. Затем происходит разрушение трехчастотного тора, и возникает сначала хаос с одним положительным и двумя нулевыми показателями Ляпунова, а далее развивается хаос с одним положительным и тремя нулевыми показателями в спектре. После этого на графике наблюдается постепенное уменьшение старших показателей Ляпунова, происходит переход к хаосу с одним положительным и двумя нулевыми показателями, и далее все пять показателей приходят к нулевому значению, при этом шестой показатель остается отрицательным (не показан на рис. 1). Таким образом, в итоге формируется пятичастотный квазипериодический режим, который и остается устойчивым при дальнейшем увеличении частотной расстройки.

В настоящей работе мы концентрируемся на спектральных свойствах наблюдаемых хаотических режимов с различной сигнатурой спектра показателей Ляпунова. Прежде всего обсудим особенности формирования хаотической динамики. Как было упомянуто ранее, при увеличении частотной расстройки происходят две седлоузловые бифуркации, в результате которых появляется трехчастотный тор. На рис. 2 представлены спектры Фурье при плавном увеличении частотной расстройки и переходе от периодических колебаний к трехчастотному тору. На рисунке линией 1 отмечен спектр Фурье для периодического режима; как и ожидалось, данный спектр представляет собой набор дискретных компонент, соответствующих основной частоте и кратным нечетным гармоникам, как это характерно для осциллятора ван дер 
Таблица 2. Точные значения пяти старших показателей Ляпунова для различных хаотических аттракторов

\begin{tabular}{l|c|l|c|c|c}
\hline$\Delta$ & $\Lambda_{1}$ & $\Lambda_{2}$ & $\Lambda_{3}$ & $\Lambda_{4}$ & $\Lambda_{5}$ \\
\hline 0.5 & 0.0075 & 0.0 & 0.0 & -0.0038 & -0.0124 \\
0.61 & 0.0045 & 0.0002 & 0.0 & -0.0001 & -0.0049 \\
1.2 & 0.0018 & 0.0 & 0.0 & 0.0 & -0.0018
\end{tabular}

Поля. При $\Delta \approx 0.1371$ в системе появляется двухчастотный тор, на графике показателей Ляпунова наблюдаются два нулевых показателя. На рис. 2 серым цветом показан пример спектра Фурье для $\Delta=0.15$, соответствующий двухчастотному тору. Как видно из рисунка, в этом случае в окрестности каждой частотной компоненты появляются компоненты-сателлиты, а также хорошо видно, что основной пик раздваивается. При дальнейшем увеличении частотной расстройки при $\Delta \approx 0.1771$ возникает трехчастотный тор, пример спектра Фурье которого показан на рис. 2 черным цветом для $\Delta=0.2$. В этом случае в окрестности компонент двухчастотного тора появляются дополнительные компоненты, что хорошо видно на увеличенном фрагменте (рис. 2, $b$ ). Таким образом, в системе в результате последовательности бифуркаций седло-узел возникает трехчастотный режим, спектр которого содержит довольно большое количество дискретных компонент.

При $\Delta \approx 0.4086$ трехчастотный тор разрушается и возникает хаос, чему соответствует появление положительной экспоненты Ляпунова в спектре. При этом в спектре показателей Ляпунова сохраняется одна дополнительная нулевая экспонента. На рис. 3, $a$ приведен пример спектра Фурье для такого динамического режима при $\Delta=0.5$, серым цветом показан спектр, а черным линия, отвечающая усреднению по 120 соседним значениям. В табл. 2 представлены значения пяти старших показателей Ляпунова. Как видно из рисунка, для хаотического сигнала спектр Фурье становится непрерывным, хотя некоторые дискретные компоненты сохраняются. В окрестности пика основной частоты, а также кратного трем и пяти формируются полосы сплошного спектра. Для основной частоты, а это частота первого генератора $f_{1}$, ширина полосы составляет около $0.2 f_{1}$, на частоте утроенной гармоники ширина полосы составила $0.7 f_{1}$, а на частоте пятой гармоники ширина полосы $-1.1 f_{1}$. Таким образом, для всех трех рабочих частотных полос можно говорить о формировании широкополосного хаотического сигнала.

При $\Delta \approx 0.5516$ второй показатель Ляпунова становится положительным и таким образом формируется гиперхаос. На рис. $3, b$ представлен пример спектра Фурье гиперхаотического режима. Форма спектра Фурье в этом случае остается такой же, следует отметить небольшое расширение полос на всех частотах и более равномерную спектральную плотность мощности.
Также рассмотрим третий вид хаотического аттрактора, который наблюдается в рассматриваемом ансамбле осцилляторов, когда в спектре показателей Ляпунова присутствуют один положительный и три нулевых показателя Ляпунова. Спектр Фурье такого динамического режима показан на рис. $3, c$. Для этого режима характерно наличие большого количества дискретных компонент в спектре, уменьшение амплитуды для всех частотных компонент.

Таким образом, показано, что в ансамбле взаимодействующих осцилляторов возможно возникновение хаотической динамики с различным спектром показателей Ляпунова в результате разрушения многочастотных квазипериодических колебаний. Хаотические колебания, возникающие таким образом, характеризуются широкополосным спектром.

\section{Финансирование работы}

Работа Е.П.С. и А.П.К. выполнена в рамках государственного задания Института радиотехники и электроники им. В.А. Котельникова РАН, работа Н.В.С. и Е.С.П. - при финансовой поддержке гранта Президента РФ для государственной поддержки молодых российских ученых МК-31.2019.8.

\section{Конфликт интересов}

Авторы заявляют, что у них нет конфликта интересов.

\section{Список литературы}

[1] Дмитриев А.С., Панас А.И. Динамический хаос: новые носители информации для систем связи. М.: Физматлит, 2002. $252 \mathrm{c}$.

[2] Короновский А.А., Москаленко О.И., Храмов А.Е. // УФН. 2009. T. 179. № 12. C. $1281-1310$.

[3] Дмитриев А.С., Ефремова Е.В., Максимов Н.А., Панас А.И. Генерация хаоса. М.: Техносфера, 2012. 424 c.

[4] Ландау Л.Д. // ДАН СССР. 1944. Т. 44. № 8. С. 339-342. [Collected Papers of L.D. Landau / Ed. D. ter Haar. Oxford: Pergamon, 1965. P. 387-391.].

[5] Станкевич Н.В., Астахов О.В., Кузнецов А.П., Селезнев Е.П. // Письма в ЖТФ. 2018. Т. 44. В. 10. С. 46-54.

[6] Kuznetsov A.P., Kuznetsov S.P., Sataev I.R., Turukina L.V. // Phys. Lett. A. 2013. V. 377. P. 3291-3295.

[7] Kuznetsov A.P., Kuznetsov S.P., Shchegoleva N.A., Stankevich N.V. // Physica D. 2019. V. 398. P. 1-12. 